\title{
Functional Analysis for Conceptual Aircraft Design
}

\begin{abstract}
To maximize the aircraft market potential, it has to be rapidly produced and made available in the market. In order to reduce time-to-market period, manufacturers need to shorten their design and development process. It becomes vital that the design architecture solution is derived faster, which can be quite handful for complex products like an aircraft with the current geometrical-based approaches due to the plethora of physical alternatives to be considered. On the other hand, the search for design architecture solution from the functional requirement is theoretically more effective since the functional space is smaller than physical search space. This allows the design efforts to be more focused and subsequently saves time, effort and resources. With this notion, there is a motivation to adapt the functional approaches into the conceptual product design process in order to exploit some of its advertised benefits. In this paper, a sample case study of an aircraft functional analysis and the discussion on how this information is useful during the conceptual design stage are presented.
\end{abstract}

Keyword: aircraft system, functional analysis, conceptual design, functional breakdown 